\title{
Electronic structure evolution of fullerene on $\mathrm{CH}_{3} \mathrm{NH}_{3} \mathrm{Pbl}_{3}$
}

\author{
Chenggong Wang, ${ }^{1}$ Congcong Wang, ${ }^{1}$ Xiaoliang Liu, ${ }^{2}$ John Kauppi, ${ }^{1}$ Yuchuan Shao, ${ }^{3}$ \\ Zhengguo Xiao, ${ }^{3}$ Cheng $\mathrm{Bi}^{3}{ }^{3}$ Jinsong Huang, ${ }^{3}$ and Yongli Gao ${ }^{1, a)}$ \\ ${ }^{1}$ Department of Physics and Astronomy, University of Rochester, Rochester, New York, 14627, USA \\ ${ }^{2}$ Hunan Key Laboratory for Super-microstructure and Ultrafast Process, College of Physics and Electronics, \\ Central South University, Changsha 410083, People's Republic of China \\ ${ }^{3}$ Department of Mechanical and Materials Engineering, Nebraska Center for Materials and Nanoscience, \\ University of Nebraska-Lincoln, Lincoln, Nebraska, 68588, USA
}

(Received 27 October 2014; accepted 24 February 2015; published online 19 March 2015)

\begin{abstract}
The thickness dependence of fullerene on $\mathrm{CH}_{3} \mathrm{NH}_{3} \mathrm{PbI}_{3}$ perovskite film surface has been investigated by using ultraviolet photoemission spectroscopy (UPS), X-ray photoemission spectroscopy (XPS), and inverse photoemission spectroscopy (IPES). The lowest unoccupied molecular orbital and highest occupied molecular orbital (HOMO) can be observed directly with IPES and UPS. It is observed that the HOMO level in fullerene shifts to lower binding energy. The XPS results show a strong initial shift of core levels to lower binding energy in the perovskite, which indicates that electrons transfer from the perovskite film to fullerene molecules. Further deposition of fullerene forms $\mathrm{C}_{60}$ solid, accompanied by the reduction of the electron transfer. The strongest electron transfer happened at 1/4 monolayer of fullerene. (C) 2015 AIP Publishing LLC.

[http://dx.doi.org/10.1063/1.4916079]
\end{abstract}

Methylammonium lead trihalides have attracted great attention as candidates for the next generation solar cells as the power conversion efficiency has exceeded $16 \% .^{1-7}$ The advantages of these materials include being solutionprocessable, low cost of large carrier mobility and long carrier lifetime, and abundance in nature. ${ }^{8-13}$ The solutionprocessibility makes it high possibility for low energy fabrication of large area photovoltaic platforms. A great amount of evidence shows that the working mechanism of the perovskite planar heterojunction (PHJ) solar cells is similar to other inorganic polycrystalline solar cells such as silicon and cadmium telluride (CdTe) materials. ${ }^{11}$ One of the most important strategies to optimize the device efficiency for those polycrystalline solar cells is to minimize the charge recombination at the surfaces and grain boundaries. Fullerene-based materials, such as fullerene $\left(\mathrm{C}_{60}\right)$ and $(6,6)$-phenyl- $\mathrm{C}_{61}$-butyric acid methyl ester (PCBM), have been the favored acceptor component in organic photovoltaic devices and recently applied in perovskite PHJ solar cells. ${ }^{14-16}$ Shao et al . showed that the fullerene layers, which were designed as electron acceptors, might effectively passivate the charge trap states at both $\mathrm{CH}_{3} \mathrm{NH}_{3} \mathrm{PbI}_{3}$ perovskite film surfaces and grain boundaries, as the fullerene eliminated the photocurrent hysteresis and doubled the efficiency of $\mathrm{CH}_{3} \mathrm{NH}_{3} \mathrm{PbI}_{3}$ solar cells. ${ }^{23}$ Given the significance of the observation, it is imperative to investigate the interface formation and energetics in order to understand the underlining physical mechanisms.

In this paper, we report our investigation on the evolution of the electronic structure and the energy level alignment at the $\mathrm{C}_{60}$ /perovskite interface using ultraviolet photoemission spectroscopy (UPS), x-ray photoemission spectroscopy (XPS), and inverse photoemission spectroscopy (IPES). We monitored the changes of the valence

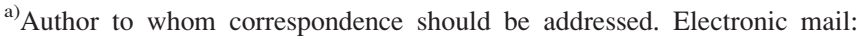
ygao@pas.rochester.edu
}

electronic structures, including the highest occupied molecular orbital (HOMO), the lowest unoccupied molecular orbital (LUMO), and the vacuum level (VL), as $\mathrm{C}_{60}$ was successively deposited layer by layer on perovskite film coated on indium tin oxide (ITO) substrate. We also monitor the core levels change by XPS. These investigations provide important insight to the improvement of the perovskite solar cells by fullerene layers.

UPS measurements were performed using a modified VG ESCA Lab system, an ultrahigh vacuum (UHV) system equipped with a He discharge lamp. The UHV system consists of three interconnecting chambers, a spectrometer chamber, an in-situ oxygen plasma (OP) treatment chamber, and an evaporation chamber with a precision air leak valve. The base pressure of the spectrometer chamber is typically $8 \times 10^{-11}$ Torr. The base pressure of the evaporation chamber is typically $1 \times 10^{-6}$ Torr. The UPS spectra were recorded by using unfiltered $\mathrm{He} \mathrm{I}(21.2 \mathrm{eV})$ excitation as the excitation source with the sample biased at $-5.00 \mathrm{~V}$ to observe the low-energy secondary cutoff. The UV light spot size on the sample is about $1 \mathrm{~mm}$ in diameter. The XPS studies were performed with a $\mathrm{MgK}_{\alpha} \mathrm{x}$-ray source $(1253.6 \mathrm{eV})$. The typical instrumental resolution for UPS and XPS measurements is 0.1 and $1.4 \mathrm{eV}$, respectively. The IPES spectra were taken with a custom-made spectrometer composed of a commercial Kimball Physics ELG-2 electron gun and a band pass photon detector prepared according to an existing design. The combined resolution (electron + photon) of the IPES spectrometer was determined to be $\sim 0.6 \mathrm{eV}$ from the Fermi edge of an evaporated Au film. All depositions were done at room temperature and monitored with a quartz crystal microbalance. For the film fabrication, $\mathrm{PbI}_{2}$ and $\mathrm{CH}_{3} \mathrm{NH}_{3} \mathrm{I}$ (MAI) were first dissolved in dimethylformamide (DMF) and 2-propanol, respectively, as precursor solutions. The precursors were then spun onto poly(3,4-ethylenedioxythiophene) poly (styrenesul-phonate) (PEDOT:PSS) covered 
ITO glass with a $\mathrm{PbI}_{2}$ layer underneath an MAI layer. A supersaturated hot solution of $\mathrm{PbI}_{2}$ was used for quick drying to obtain a pin-hole free and conformal $\mathrm{PbI}_{2}$ layer on the PEDOT:PSS surface. Since $\mathrm{PbI}_{2}$ has relatively low solubility in 2-propanol, the spin coating of MAI solution did not wash off the $\mathrm{PbI}_{2}$. The bilayer film was then annealed at a temperature of $100{ }^{\circ} \mathrm{C}$ for $120 \mathrm{~min}$.

Thermal admittance spectroscopy (TAS) analysis was used to quantize the reduction of trap states in perovskite films by the passivation of $\mathrm{C}_{60}$ and PCBM. TAS is a wellestablished technique for charactering both shallow and deep defect levels. TAS has been widely applied in understanding defects in thin film solar cell ${ }^{17}$ and organic solar cells. ${ }^{18}$ Shao et al demonstrated that there was a relatively large density of defect states on the order of $1 \times 10^{17}$ to $1 \times 10^{19} \mathrm{~m}^{-3}$ in the devices without any fullerene passivation, which indicated the large hysteresis of photocurrents observed. ${ }^{23}$ The trap density of state (tDOS) with an energy level above $0.40 \mathrm{eV}$ decreased by one order of magnitude just after the spin coating of $\mathrm{C}_{60}$ on the perovskite films even without thermal annealing. Especially with the energy level above $0.5 \mathrm{eV}$, the tDOS decreased for nearly two orders. The remarkable decrease of the tDOS was consistent with the decreased photocurrent hysteresis, indicating the effective passivation of charge traps in perovskite by $\mathrm{C}_{60}$. But the charge transfer situation is not clear at the interface of $\mathrm{C}_{60}$ and perovskite. In order to investigate the charge transfer process between the $\mathrm{C}_{60}$ and perovskite, we carried out the layer by layer research of $\mathrm{C}_{60}$ on perovskite.

In Figure 1, we show the UPS and IPES spectrum of the perovskite film. The UPS spectrum was taken first and then the IPES spectrum was recorded on the same film subsequently. The origin in Figure 1 is the Fermi level, which was calibrated by UPS and IPES on the Au/Si substrates. The spectra on the left side of the Fermi level are the valence bands from UPS, while the spectra on the right side are the conduction bands from IPES. For the pristine perovskite film, the valence band (VB) feature is very clear and the valence band maximum (VBM) is $0.76 \mathrm{eV}$. The conduction band minimum $(\mathrm{CBM})$ is $-0.90 \mathrm{eV}$, which is consistent with the previous reports. ${ }^{3,19} \mathrm{We}$ define the onset as the extrapolation of the leading edge (closer to the Fermi level) in the

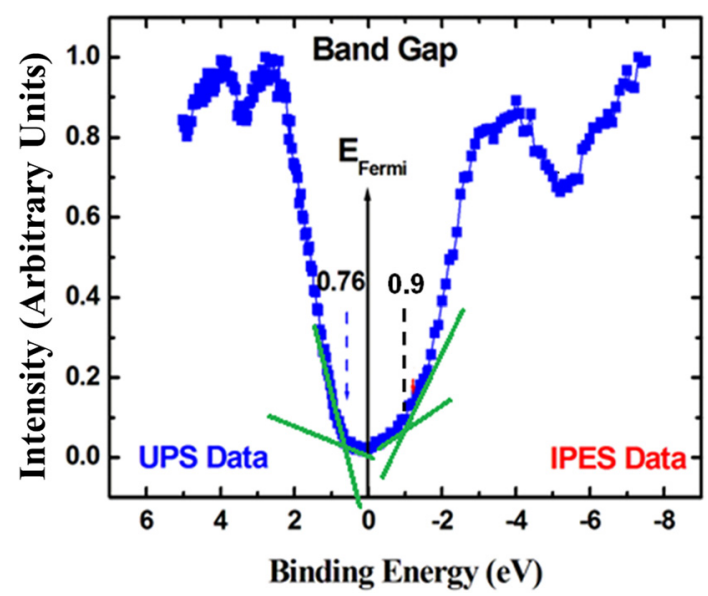

FIG. 1. UPS and IPES spectra of the perovskite film. spectrum. The results are an injection energy gap of about $1.66 \mathrm{eV}$, interestingly very close to the previous report. ${ }^{19,20}$

In Figure 2, the evolution of UPS spectra due to different $\mathrm{C}_{60}$ thickness is presented. $\mathrm{C}_{60}(99.99 \%$ purity) was purchased from SES Research and thermally deposited on the perovskite film. Figure 2(a) shows the cutoff changes of the UPS spectra, which reflect the vacuum-level change of the samples. It is very impressive that a very thin layer of $\mathrm{C}_{60}$ film (as low as $2 \AA$ ) can significantly change the vacuum level, thus the sample work function. The pristine perovskite film had a WF of $4.73 \mathrm{eV}$, which is consistent with the previous reports. ${ }^{21}$ With the $\mathrm{C}_{60}$ thickness increasing, the WF shifted to lower binding energy, which means higher WF. The WF was $6.15 \mathrm{eV}$ when the $\mathrm{C}_{60}$ thickness was at $2 \AA$. The change slowed down and finally saturated when the $\mathrm{C}_{60}$ thickness reached to $128 \AA$. In Figure 2(b), it shows the HOMO region of the UPS spectra of the same films. It can be observed that with the $\mathrm{C}_{60}$ thickness increasing, the HOMO shift followed the cutoff changes, implying that the $\mathrm{C}_{60}$ molecule does not undergo significant chemical modification. No new state emerges from the gap with the $\mathrm{C}_{60}$ thickness increasing. The shifts of the energy levels were due to the charge transfer from the perovskite films to $\mathrm{C}_{60}$ molecules.

To further reveal the thickness dependence and charge transfer process, we performed detailed XPS core level analysis. Figure 3 shows the XPS data of C 1s, O 1s, I 3d, and Pb $4 \mathrm{f}$ core levels with the different $\mathrm{C}_{60}$ thickness. All the spectra have been normalized to the same height for visual clarity. In Figure 3(a), we observed the core levels for $\mathrm{C}$ 1s. There was a strong initial shift to lower binding energy when $2 \AA$ $\mathrm{C}_{60}$ deposited on the perovskite film. With the $\mathrm{C}_{60}$ thickness increasing, the shift trend to the lower binding energy slowed down and saturated when the thickness reached to $128 \AA$. Besides that of $\mathrm{C}$, all the other core levels are attenuated as the $\mathrm{C}_{60}$ layer thickness increases, especially, the $\mathrm{O} 1 \mathrm{~s}$ signal because it is weak as it comes from the oxygen contamination from the air.

In order to elucidate the XPS core level shifts more clearly, we show the energy level shift versus $\mathrm{C}_{60}$ thickness in Figure 4. The inset is the amplified region for $2-16 \AA \mathrm{C}_{60}$.

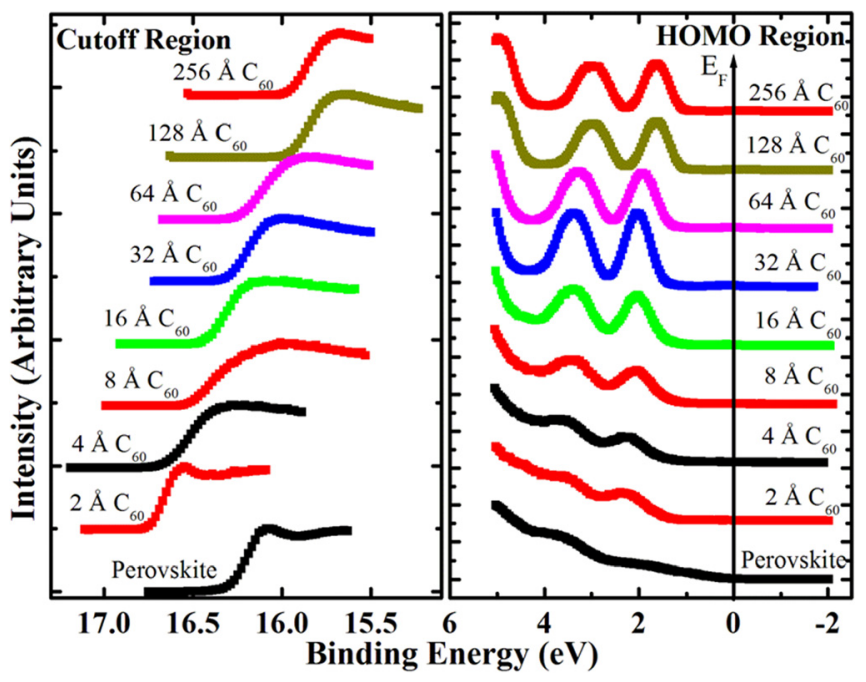

FIG. 2. UPS spectra evolution of $\mathrm{C}_{60}$ film on the perovskite: (a) cutoff and (b) HOMO region. Saturation of the energy level shift at $128 \AA \mathrm{C}_{60}$ is evident. 


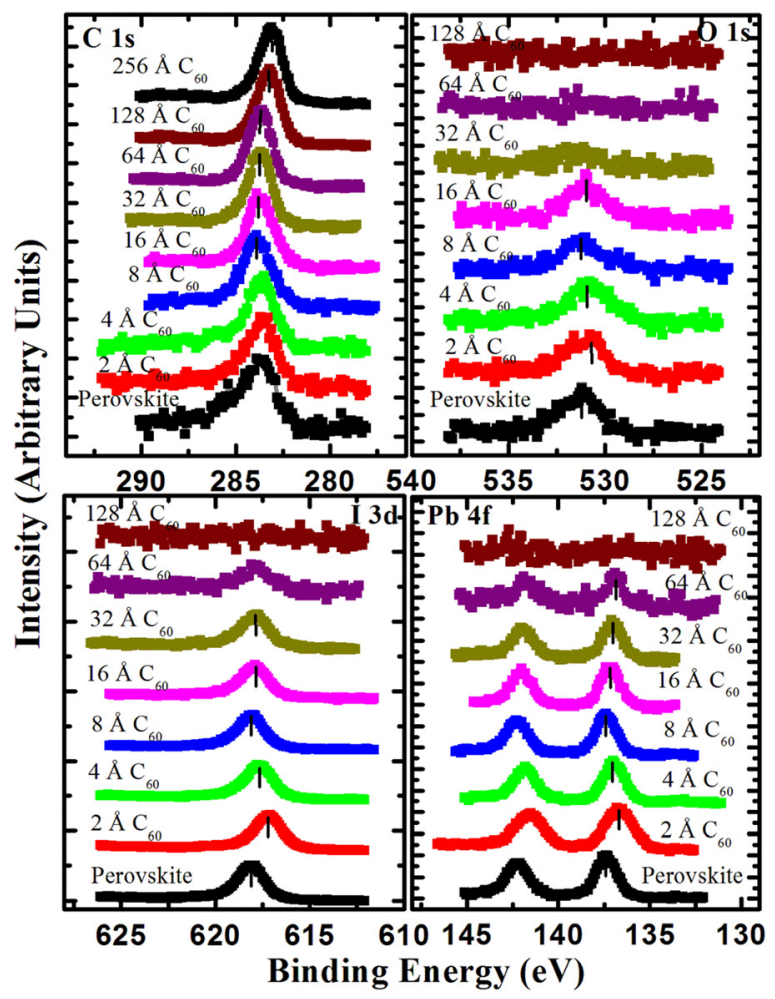

FIG. 3. XPS spectra of (a) C 1s, (b) O 1s, (c) I 3d, and (d) Pb 4 f core levels at different thickness of $\mathrm{C}_{60}$.

We observed the strong initial shift to lower binding energy when $2 \AA \mathrm{C}_{60}$ was deposited. The energy shift is about $0.3 \mathrm{eV}$. All other XPS spectra shifted to the lower binding energy as well. Then the core levels gradually shifted to higher binding energy for all the XPS spectra. The shifts for $\mathrm{C} 1 \mathrm{~s}$ and $\mathrm{O} 1 \mathrm{~s}$ are smaller than the shifts of $\mathrm{N} 1 \mathrm{~s}, \mathrm{~Pb} 4 \mathrm{f}$, and I $3 \mathrm{~d}$. It is not surprising, as the $\mathrm{C} 1 \mathrm{~s}$ has increasing contribution from $\mathrm{C}_{60}$, and $\mathrm{O}$ is from the air contamination. When 8 $\AA \mathrm{C}_{60}$ was deposited, the core levels began to shift to lower binding energies and finally saturated at $128 \AA$ of $\mathrm{C}_{60}$. For all the spectra with different thickness of $\mathrm{C}_{60}$, we observe the same trend of shift. We could draw a conclusion that the

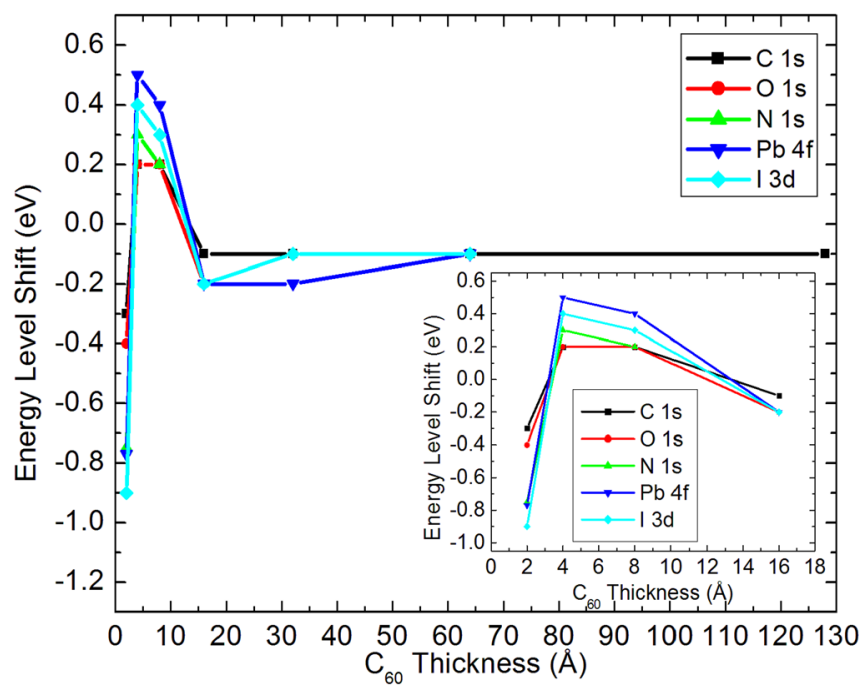

FIG. 4. Energy level shifts versus the $\mathrm{C}_{60}$ thickness. The inset shows the amplified region for $2-16 \AA \mathrm{C}_{60}$. strong initial shift to the lower binding energy for all spectra indicated the electron transfer from the perovskite to the $\mathrm{C}_{60}$ molecule. Further deposition of $\mathrm{C}_{60}$ forms $\mathrm{C}_{60}$ solid, which results in a reduction of the electron transfer. The UPS results showed that with the $\mathrm{C}_{60}$ thickness increasing, the WF and HOMO levels shifted to the lower binding energy. Our XPS results have the same trend with the $\mathrm{C}_{60}$ thickness increase, especially when the thickness is over $8 \AA$, as the interface charge $\mathrm{C}_{60}$ electron transfer has completed and a rigid shift in the $\mathrm{C}_{60}$ is taking place.

The energy level diagram for $256 \AA \mathrm{C}_{60}$ /perovskite interface is depicted in Figure 5. In Figure 5, the interface between perovskite and $\mathrm{C}_{60}$ has an interface dipole of $0.5 \mathrm{eV}$ and $\mathrm{HOMO}$ onset $\mathrm{BE}$ of perovskite was measured to be $0.6 \mathrm{eV}$. The $\mathrm{WF}$ of the perovskite was measured to be $5.0 \mathrm{eV}$. Near the interface between perovskite and $\mathrm{C}_{60}$, the WF and VBM level were lifted up by $0.1 \mathrm{eV}$, which indicated the interface dipole as $0.5 \mathrm{eV}$. There is a clear band bending at the $\mathrm{C}_{60}$ region. The $\mathrm{HOMO}$ onset of $\mathrm{C}_{60}$ was measured to be $1.86 \mathrm{eV}$. With further $\mathrm{C}_{60}$ deposition a gradual relaxation was observed, resulting in a band bending region of $\sim 200 \AA$. The energy level alignment indicated electron transfer from the perovskite to $\mathrm{C}_{60}$ molecule. These are high energy level electrons that may have acted as charge recombination/scattering centers, and removing them from the perovskite should contribute to the reduction in trap states observed in the device studies. With the $\mathrm{C}_{60}$ thickness increasing, the electron transfer reduced and finally saturated when the $\mathrm{C}_{60}$ thickness reached to $128 \AA$. We also compared the $\mathrm{C}_{60}$ band structures with the previous report. ${ }^{22}$ We notice that the pristine $\mathrm{C}_{60}$ has the HOMO and LUMO levels at $1.38 \mathrm{eV}$ and $-0.92 \mathrm{eV}$, respectively. In this case, we discovered that the $\mathrm{C}_{60}$ was n-type at the interface and became more neutral away from the interface as the HOMO level was pulled up and got closer to the Fermi level. Shao et al. also indicated that with thermal annealing process, the density of relatively shallow defect states reduced gradually, while there is rarely further reduction of deep trap density. It is possible that thermal annealing may drive $\mathrm{C}_{60}$ into the grain boundaries as individual molecules that act as strong electron absorbers as the $1 / 4$ monolayer case, and further reduce the density of

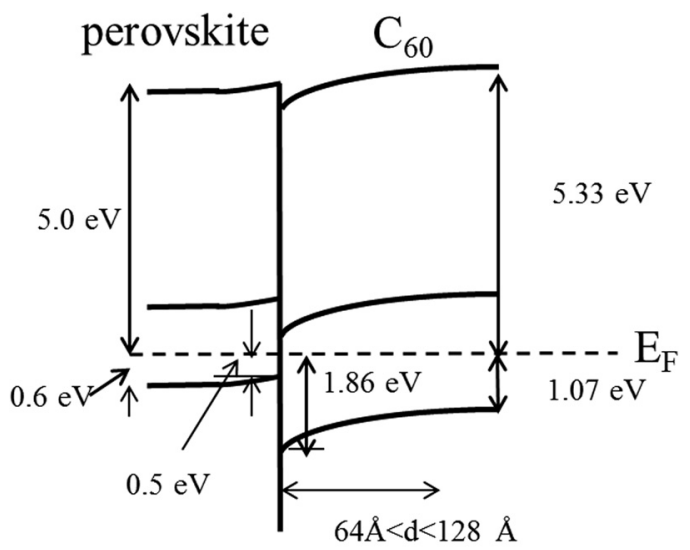

Interface dipole $0.5 \mathrm{eV}$

FIG. 5. Energy level alignment at $256 \AA \mathrm{C}_{60}$ /perovskite. 
defect states. We speculated the shallow trap states stay deeper in the perovskite films, such as grain boundaries inside the perovskite layer, which can only be passivated by the diffusion of $\mathrm{C}_{60}$ into the perovskite layers.

In conclusion, we have measured the electronic structure evolution of $\mathrm{C}_{60}$ on perovskite. We observed that the WF of the perovskite is $5.0 \mathrm{eV}$ and the $\mathrm{VBM}$ is $0.6 \mathrm{eV}$. The band gap of the perovskite is $1.66 \mathrm{eV}$, which is in accordance with the previous reports. There is an interface dipole as $0.5 \mathrm{eV}$ at the interface of perovskite and $\mathrm{C}_{60}$. We also observed the HOMO level of $\mathrm{C}_{60}$ shifts to lower binding energy, indicating a band bending in the $\mathrm{C}_{60}$ region. The perovskite core levels show a strong initial shift to lower binding energy, indicating electron transfer from the perovskite film to fullerene molecules. The strongest electron transfer happened at $1 / 4$ monolayer of fullerene, and further deposition reduced the transfer as forms fullerene forms $\mathrm{C}_{60}$ solid film.

This work was supported in part by the National Science Foundation (Grant Nos. NSF CBET-1437656 and DMR1303742) and the National Natural Science Foundation of China (Grant Nos. 51203192, 51173205, and 11334014) and from the Natural Science Foundation of Hunan Province, China (Grant No. 12JJ3003). J. Huang thanks the financial supported from U.S. Department of Energy under Award No. DE-EE0006709 for the perovskite material preparation and data analysis.

${ }^{1}$ G. Hodes and D. Cahen, Nat. Photonics 8(2), 87-88 (2014).

${ }^{2}$ G. Hodes, Science 342(6156), 317-318 (2013).

${ }^{3}$ H.-S. Kim, C.-R. Lee, J.-H. Im, K.-B. Lee, T. Moehl, A. Marchioro, S.-J. Moon, R. Humphry-Baker, J.-H. Yum, and J. E. Moser, Sci. Rep. 2, 591 (2012).

${ }^{4}$ M. M. Lee, J. Teuscher, T. Miyasaka, T. N. Murakami, and H. J. Snaith, Science 338(6107), 643-647 (2012).
${ }^{5}$ M. Liu, M. B. Johnston, and H. J. Snaith, Nature 501(7467), 395-398 (2013).

${ }^{6}$ A. Abrusci, S. D. Stranks, P. Docampo, H.-L. Yip, A. K.-Y. Jen, and H. J. Snaith, Nano Lett. 13(7), 3124-3128 (2013).

${ }^{7}$ S. D. Stranks, G. E. Eperon, G. Grancini, C. Menelaou, M. J. P. Alcocer, T. Leijtens, L. M. Herz, A. Petrozza, and H. J. Snaith, Science 342(6156), 341-344 (2013).

${ }^{8}$ T. Baikie, Y. N. Fang, J. M. Kadro, M. Schreyer, F. X. Wei, S. G. Mhaisalkar, M. Graetzel, and T. J. White, J. Mater. Chem. A 1(18), 5628-5641 (2013).

${ }^{9}$ B. Conings, L. Baeten, C. De Dobbelaere, J. D'Haen, J. Manca, and H. G. Boyen, Adv. Mater. 26(13), 2041-2046 (2014).

${ }^{10}$ C. Wehrenfennig, G. E. Eperon, M. B. Johnston, H. J. Snaith, and L. M. Herz, Adv. Mater. 26(10), 1584-1589 (2014).

${ }^{11}$ G. Xing, N. Mathews, S. S. Lim, N. Yantara, X. Liu, D. Sabba, M. Grätzel, S. Mhaisalkar, and T. C. Sum, Nat. Mater. 13(5), 476-480 (2014).

${ }^{12}$ E. Edri, S. Kirmayer, S. Mukhopadhyay, K. Gartsman, G. Hodes, and D. Cahen, Nat. Commun. 5, 3461 (2014).

${ }^{13}$ Z. Xiao, C. Bi, Y. Shao, Q. Dong, Q. Wang, Y. Yuan, C. Wang, Y. Gao, and J. Huang, Energy Environ. Sci. 7, 2619 (2014).

${ }^{14}$ Y. Matsuo, J. Hatano, T. Kuwabara, and K. Takahashi, Appl. Phys. Lett. 100(6), 063303 (2012).

${ }^{15}$ R. B. Ross, C. M. Cardona, D. M. Guldi, S. G. Sankaranarayanan, M. O. Reese, N. Kopidakis, J. Peet, B. Walker, G. C. Bazan, and E. Van Keuren, Nat. Mater. 8(3), 208-212 (2009).

${ }^{16}$ C. J. Brabec, S. Gowrisanker, J. J. Halls, D. Laird, S. Jia, and S. P. Williams, Adv. Mater. 22(34), 3839-3856 (2010).

${ }^{17}$ T. Walter, R. Herberholz, C. Muller, and H. W. Schock, J. Appl. Phys. 80(8), 4411-4420 (1996).

${ }^{18}$ C. Melzer, E. J. Koop, V. D. Mihailetchi, and P. W. Blom, Adv. Funct. Mater. 14(9), 865-870 (2004).

${ }^{19}$ G. Xing, N. Mathews, S. Sun, S. S. Lim, Y. M. Lam, M. Grätzel, S. Mhaisalkar, and T. C. Sum, Science 342(6156), 344-347 (2013).

${ }^{20}$ H.-S. Kim, J.-W. Lee, N. Yantara, P. P. Boix, S. A. Kulkarni, S. Mhaisalkar, M. Grätzel, and N.-G. Park, Nano Lett. 13(6), 2412-2417 (2013).

${ }^{21}$ A. Kojima, K. Teshima, Y. Shirai, and T. Miyasaka, J. Am. Chem. Soc. 131(17), 6050-6051 (2009).

${ }^{22}$ Irfan, M. Zhang, H. Ding, C. W. Tang, and Y. Gao, Org. Electron. 12(9), 1588-1593 (2011).

${ }^{23}$ Y. Shao, Z. Xiao, C. Bi, Y. Yuan, and J. Huang, Nat. Commun. 5, A 5784 (2015). 